\title{
A brief nap is beneficial for human route-learning: The role of navigation experience and EEG spectral power
}

\author{
Erin J. Wamsley, ${ }^{1}$ Matthew A. Tucker, Jessica D. Payne, and Robert Stickgold \\ Center for Sleep and Cognition, Beth Israel Deaconess Medical Center, Harvard Medical School, Boston, Massachusetts 02215, USA
}

\begin{abstract}
Here, we examined the effect of a daytime nap on changes in virtual maze performance across a single day. Participants either took a short nap or remained awake following training on a virtual maze task. Post-training sleep provided a clear performance benefit at later retest, but only for those participants with prior experience navigating in a three-dimensional (3D) environment. Performance improvements in experienced players were correlated with delta-rich stage 2 sleep. Complementing observations that learning-related brain activity is reiterated during post-navigation NREM sleep in rodents, the present data demonstrate that NREM sleep confers a performance advantage for spatial memory in humans.
\end{abstract}

[Supplemental material is available online at http://www.learnmem.org.]

A growing body of animal and human literature suggests that the consolidation of memories occurs optimally during periods of post-learning sleep. Nonrapid eye movement sleep (NREM), in particular, may be beneficial for the offline consolidation of hippocampus-dependent learning. The neurophysiological basis for this hypothesis is derived largely from electrophysiological studies in rodents, demonstrating that patterns of hippocampal place cell activity first seen during waking exploration are later reexpressed during post-learning sleep (Wilson and McNaughton 1994; Kudrimoti et al. 1999; Nadasdy et al. 1999; Ji and Wilson 2007). Behavioral studies in humans meanwhile demonstrate that NREM sleep is beneficial for declarative memory performance, relative to equivalent periods of wakefulness (Plihal and Born 1997; Tucker et al. 2006). However, the memory tasks typically employed in human research are quite different from those used in rodents, with human studies most often focusing on the memorization of verbal or visual stimuli (Plihal and Born 1997; Schabus et al. 2004; Clemens et al. 2005; Ellenbogen et al. 2006; Tucker et al. 2006; Daurat et al. 2008). Thus far, sleep-dependent memory reactivation has not been established to be directly beneficial for memory performance in an animal model, as the protocols employed in this research typically involve welllearned simple tasks which do not easily lend themselves to measurement of learning across time (Wilson and McNaughton 1994; Kudrimoti et al. 1999). Although the hippocampal memory reactivation described in rodents is a possible explanation for the effect of NREM sleep on human declarative memory, widely divergent methodologies employed across species prohibit confidence in this conclusion.

Bridging this conceptual gap, a small handful of studies have begun to explore the relationship between spatial navigation and NREM sleep in humans. Notably, a PET study by Peigneux et al. (2004) demonstrated that learning-related hippocampal activity seen while training on a virtual maze task is again expressed during post-learning human sleep. Furthermore, this hippocampal reactivation strongly predicted overnight improvement on the

'Corresponding author.

E-mail EWamsley@bidmc.harvard.edu; fax (617) 667-8498.

Article is online at http://www.learnmem.org/cgi/doi/10.1101//m.1828310. task (Peigneux et al. 2004). Additional studies have suggested a link between sleep and other types of spatial-related learning, including mental rotation performance (Plihal and Born 1999), the ability to reproduce a complex figure (Clemens et al. 2006; Tucker and Fishbein 2008), performance on a computerized version of Milner's (1965) "bolt head" maze (Tucker and Fishbein 2008), and memory for the location of verbal information on a screen (Daurat et al. 2008).

Yet it remains unclear whether sleep, relative to wakefulness, provides a performance benefit for human route-learning in the context of a realistic spatial environment. Navigation through virtual environments is a strongly hippocampus-dependent task (Peigneux et al. 2004; Astur et al. 2005) and provides an experimental model closely paralleling the spatial exploration tasks employed in the rodent literature. However, the few studies reporting effects of sleep on human navigation performance have been contradictory. Using a navigation task similar to that of Peingeux et al. (2004), Orban et al. (2006) failed to detect any effect of post-learning sleep deprivation on maze performance but did find evidence of altered task-related brain activity, concluding that sleep supports "covert" memory reorganization (Orban et al. 2006). In direct contrast, Ferrara et al. found that spatial memory is improved when a retention interval falls across a night of sleep, relative to when route memory must be retained during daytime wakefulness, or across a night of sleep deprivation (Ferrara et al. 2006, 2008).

The present study clarifies these issues by examining the effect of a post-learning nap on complex route-learning in a threedimensional (3D) virtual environment. When controls are tested at a different time of day than sleep participants, circadian confounds may present a substantial problem. Alternatively, overnight protocols employing sleep-deprived subjects necessarily suffer from confounds related to this sleep deprivation during the retention interval. The use of a daytime nap as a sleep intervention avoids these pitfalls by allowing all subjects to be trained and tested at the same circadian time, and in the absence of sleep deprivation. A series of recent studies confirm that a daytime nap is sufficient to induce performance improvements on declarative and procedural memory tasks, relative to wake subjects (Mednick et al. 2003; Backhaus and Junghanns 2006; Nishida and 
Table 1. Sleep architecture during the nap period (mean \pm SD)

\begin{tabular}{lcc}
\hline & Novice players & $\begin{array}{c}\text { Experienced } \\
\text { players }\end{array}$ \\
\hline TST $^{\text {a }}$ & $39.29 \pm 11.40$ & $49.72 \pm 11.06$ \\
Stage 1 (min) & $9.79 \pm 2.58$ & $9.28 \pm 2.58$ \\
Stage 1 (\%) & $27.27 \pm 14.54$ & $19.18 \pm 10.75$ \\
Stage 2 (min) & $26.21 \pm 12.06$ & $29.31 \pm 8.97$ \\
Stage 2 (\%) & $64.87 \pm 14.45$ & $59.17 \pm 14.68$ \\
SWS (min) & $3.29 \pm 5.87$ & $9.47 \pm 11.49$ \\
SWS (\%) & $8.34 \pm 14.35$ & $18.44 \pm 21.83$ \\
REM (min) & $0.00 \pm 0.00$ & $1.16 \pm 3.03$ \\
REM (\%) & $0.00 \pm 0.00$ & $2.24 \pm 5.89$ \\
\hline
\end{tabular}

${ }^{a}$ There were no significant differences between groups on any measure, but there was a trend for total sleep time (TST) to be greater in experienced players $(P=0.052)$.

Means \pm SD. SWS, slow wave sleep stages 3 and 4 . \%, Percent of TST. Of the nap participants, $n=12$ did not enter SWS during the sleep period, and $n=$ 3 were awoken from REM sleep. Due to artifact, the sleep recording for one novice player was unusable.

Walker 2006; Tucker et al. 2006; Lahl et al. 2008; Tucker and Fishbein 2008).

Participants ( $n=53,34$ female) were trained on a virtual maze-learning task at 12:30 pm. Following training, nap participants lay down for a 1.5 -h sleep opportunity. These subjects were allowed to obtain as much NREM sleep as possible but were awoken at the first signs of REM (see Table 1 for sleep data). Wake subjects meanwhile began the retention interval with a 20-min period of "quiet wakefulness," during which they sat silently, not engaged in any particular activity. Wake subjects were then allowed to watch videos for the remainder of the intervention period, in order to assist in wake maintenance. Characteristics of nap and wake participant groups are described in Supplemental Table S1. All subjects were retested on the maze navigation task following a delay, at 5:30 pm.

The virtual maze task was a simple 3D environment designed for this research (Fig. 1; see also Supplemental Methods). In brief, subjects initially spent 5 min exploring a complex maze and were instructed to remember the layout of the maze environment as well as possible. Subsequently, subjects navigated through the same maze during three test trials, in which they were instructed to reach a specified goal point as quickly as possible. Performance

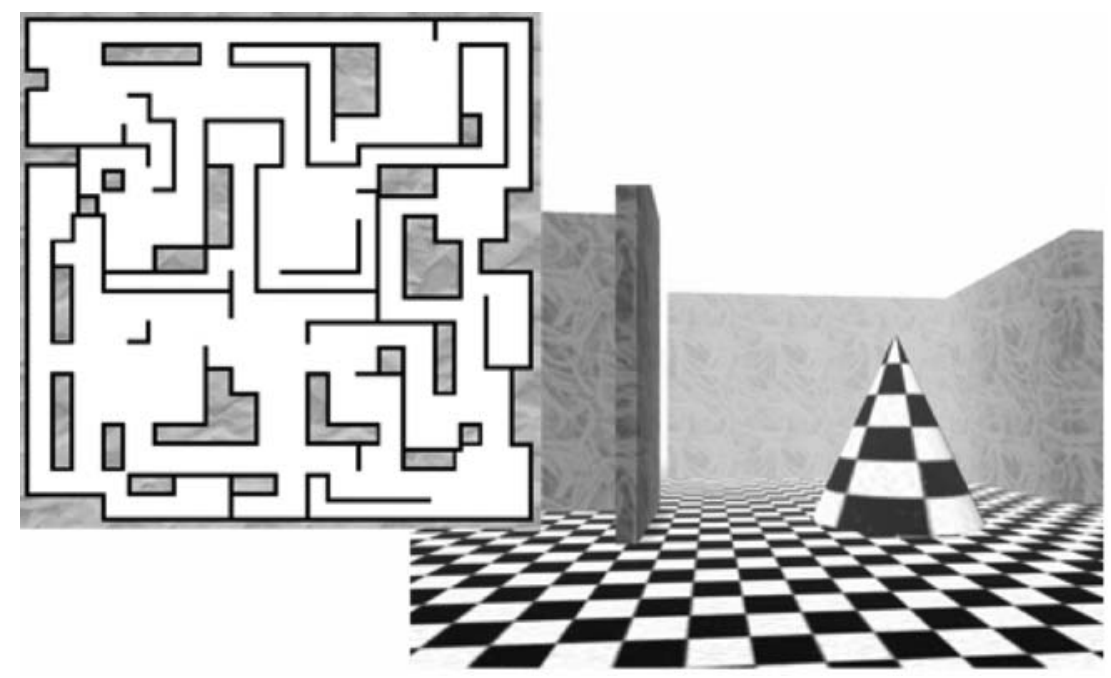

Figure 1. A sample screen from one location within the maze, as seen by the subject, displayed alongside a bird's-eye view layout of difficulty level 3 . was assessed as time required to reach the goal on each trial, and improvement was calculated as the change in performance from the last training trial (trial 3), to mean performance on the three retest trials (trials 4-6, administered at 5:30 pm). All subjects rated their prior experience with 3D-style game environments on a five-point scale, on which they assessed their typical frequency of play ranging from "every day" to "less than once per year."

We hypothesized that post-learning sleep would lead to enhanced retest performance on this hippocampus-dependent spatial task. Furthermore, we expected that sleep-dependent performance improvements would correlate with spectral power in low-frequency EEG bands during the nap $(<1 \mathrm{~Hz}$ slow oscillation and/or 1-4 Hz delta power).

Maze performance improved significantly across the six training and retest trials $\left(F_{(5,230)}=2.35, P=0.04, \eta_{\mathrm{p}}^{2}=0.05\right)$. Overall, performance changes across the retention interval did not differ significantly between nap and wake subjects (for raw improvement: $t_{(46)}=1.22, P>0.2$; percentage improvement: $\left.t_{(46)}=1.5, P>0.1\right)$. We observed, however, that baseline performance on the final training trial was strongly dependent on prior experience with 3D games, as self-assessed on a five-point scale $\left(F_{(4,43)}=4.92, P=0.002\right.$; see Supplemental Methods). Prior research suggests that individuals who perform poorly on learning tasks prior to sleep fail to exhibit sleep-dependent performance improvements (Tucker and Fishbein 2008). We therefore investigated whether the effect of sleep on maze performance might be mediated by subjects' virtual navigation experience. Post-hoc tests (Tukey's HSD) revealed that only subjects at the bottom of the experience scale (no prior game experience or less than once per year) differed at baseline from subjects at other experience levels (Supplemental Fig. S1). The sample was therefore split into novice $(n=16$, experience less than once per year; mean time to complete last training trial $=421 \mathrm{sec} \pm 209 \mathrm{SD}$ ) and experienced players $(n=32$, experience equal to or greater than once per year; mean $=184 \mathrm{sec} \pm 150 ; t_{(46)}=4.5, P<0.001, d=1.3$; see Table 2 and Supplemental Fig. S1).

Sleep imparted a performance benefit relative to wake exclusively for experienced game players. A $2 \times 2$ ANOVA on changes in maze performance across the day revealed an interaction between prior game experience and sleep condition (raw improvement: $F_{(1,44)}=5.6, P=0.02, \eta_{\mathrm{p}}^{2}=0.12$; percent improvement: $F_{(1,44)}=$ 3.7, $P=0.06, \eta_{\mathrm{p}}^{2}=0.08$; see Fig. 2$)$. In experienced players, postlearning sleep provided a performance benefit relative to wakefulness, whether measured as raw $\left(t_{(30)}=2.5, P=0.01\right)$ or percentage improvement $\left(t_{(30)}=2.1\right.$, $P=0.04)$. While the performance of experienced gamers deteriorated across wakefulness (raw improvement, $P=$ 0.05 ; percent improvement, $P=0.02$ ), there was no significant change in performance across the nap (Fig. 2, top). However, stage 2 delta power $(1-4 \mathrm{~Hz})$ strongly predicted the presence and extent of post-nap improvement (percentage improvement: $r_{16}=0.49, P=$ 0.05; raw improvement: $r_{16}=0.61, P=$ 0.01; Fig. 3, top). In fact, those subjects with the greatest stage 2 delta power actually exhibited quite large sleepdependent improvements (Fig. 3, top). As might be expected from the reciprocal relationship between delta power and spindle activity (De Gennaro and Ferrara 2003), raw performance improvement in experienced players was 
Table 2. Characteristics of novice and experienced players (mean \pm SD)

\begin{tabular}{|c|c|c|c|}
\hline & $\begin{array}{l}\text { Novice players } \\
\quad(n=16)\end{array}$ & $\begin{array}{c}\text { Experienced } \\
\text { players }(n=32)\end{array}$ & $P$-value \\
\hline Experience $\mathrm{w} /$ first-person games $(0-4)$ & $0.00( \pm 0.00)$ & $2.03( \pm 1.03)$ & $<0.001^{\mathrm{a}}$ \\
\hline Age & $22.81( \pm 3.27)$ & $21.16( \pm 2.83)$ & $>0.3$ \\
\hline Percent female & $56.25 \%$ & $18.75 \%$ & $<0.1$ \\
\hline Maze difficulty level assigned $(1-4)$ & $2.75( \pm 0.86)$ & $3.3( \pm 0.97)$ & $<0.1$ \\
\hline $\begin{array}{l}\text { Baseline performance (last training } \\
\text { trial performance) }\end{array}$ & $420.69( \pm 208.52)$ & $184.25( \pm 149.93)$ & $<0.001^{\mathrm{a}}$ \\
\hline Task difficulty VAS ${ }^{\mathrm{b}}(0-8)$ & $3.04( \pm 1.17)$ & $3.31( \pm 1.55)$ & $>0.5$ \\
\hline Task engagement VAS (0-8) & $3.61( \pm 2.31)$ & $4.61( \pm 1.53)$ & $<0.1$ \\
\hline Mean bedtime from log & $12: 40( \pm 74 \mathrm{~min})$ & $12: 38( \pm 55 \mathrm{~min})$ & $>0.9$ \\
\hline Mean wake time from log & $8: 31( \pm 69 \mathrm{~min})$ & $8: 26( \pm 44 \mathrm{~min})$ & $>0.7$ \\
\hline Training phase $\mathrm{SSS}^{\mathrm{C}}$ & $2.63( \pm 0.80)$ & $2.75( \pm 0.95)$ & $>0.6$ \\
\hline Retest SSS & $2.47( \pm 0.92)$ & $2.47( \pm 1.14)$ & $>0.9$ \\
\hline
\end{tabular}

${ }^{a}$ Other than game experience, novice and experienced participants differed significantly only in terms of baseline performance. Maze difficulty level did not significantly predict either raw improvement $(P>0.6)$ or percentage improvement $(P>0.2)$ in completion times, and inclusion of this variable as a covariate in primary analyses of the sleep effect did not alter the outcome of these analyses (see Supplemental Results). Means \pm SD.

${ }^{\mathrm{b}} \mathrm{VAS}=$ Visual Analog Scale.

${ }^{c}$ SSS $=$ Stanford Sleepiness Scale.

negatively correlated with power in the spindle band during stage 2 sleep $\left(11-15 \mathrm{~Hz} ; r_{16}=-0.57, P=0.02\right)$. Percentage improvement was unrelated to spindle power. For further detail on EEG analyses, see Supplemental Methods.

Baseline maze performance (time to complete last training trial) was also correlated with stage 2 delta power during the nap $\left(r_{16}=0.71, P=0.002\right.$; Fig. 3 , bottom $)$ and predicted subsequent improvement. However, it is critical to note that baseline score predicted performance improvements on the maze selectively within the nap group (correlation with raw improvement: $r_{16}=0.85$, $P<0.001$; percentage improvement: $\left.r_{16}=0.67, P=0.005\right)$. That a similar relationship was not seen in wake subjects suggests sleep-dependent processes were required for this correlation to emerge. After correction for multiple comparisons (significance threshold set to $P=$ 0.02 based on a modified Bonferroni correction, see Supplemental Methods), significant correlations between delta power and baseline performance were observed exclusively over left central/ parietal sites, whereas the aforementioned correlations between delta power and performance improvements were observed predominantly over central electrodes (see Fig. 3).

Novice game players exhibited substantial performance improvements at retest (raw improvement: $t_{(15)}=3.17$, $P=0.006, d=1.18$; percentage improvement: $t_{(15)}=3.33, P=0.005, d=1.50$; Fig. 2, bottom) but did not benefit from post-learning sleep $(P>0.2$ for both raw and percentage improvement measures). In contrast to experienced players, in novices, neither baseline performance
$(P=0.2)$ nor performance improvements across the day (raw improvement: $P>0.9$; percent improvement: $P>0.7$ ) were related to delta power during the nap. In novice, as well as in experienced players, sleep architecture variables (TST, time in SWS, time in stage 2, time in stage 1 , and time in REM) were unrelated to performance improvements across the day and were unrelated to baseline performance levels.

Numerous animal studies have now demonstrated that following performance of spatial tasks, explorationrelated brain activity is reexpressed during NREM sleep. The present findings suggest that NREM sleep supports the consolidation of spatial memory in humans. We examined the effect of a daytime nap on changes in virtual maze performance across the day. As hypothesized, post-learning NREM sleep imparted a benefit for maze performance at later retest, relative to a period of wakefulness. Interestingly, sleep only provided this benefit for participants with greater prior experience in navigating through 3D-style virtual environments. These experienced game players performed well at baseline and improved their performance across the course of training. A brief nap on average served to stabilize memory performance in these experienced subjects, with enhancement of memory performance occurring only if the post-learning nap was rich in delta activity. Meanwhile, an equal period spent awake resulted in substantial performance
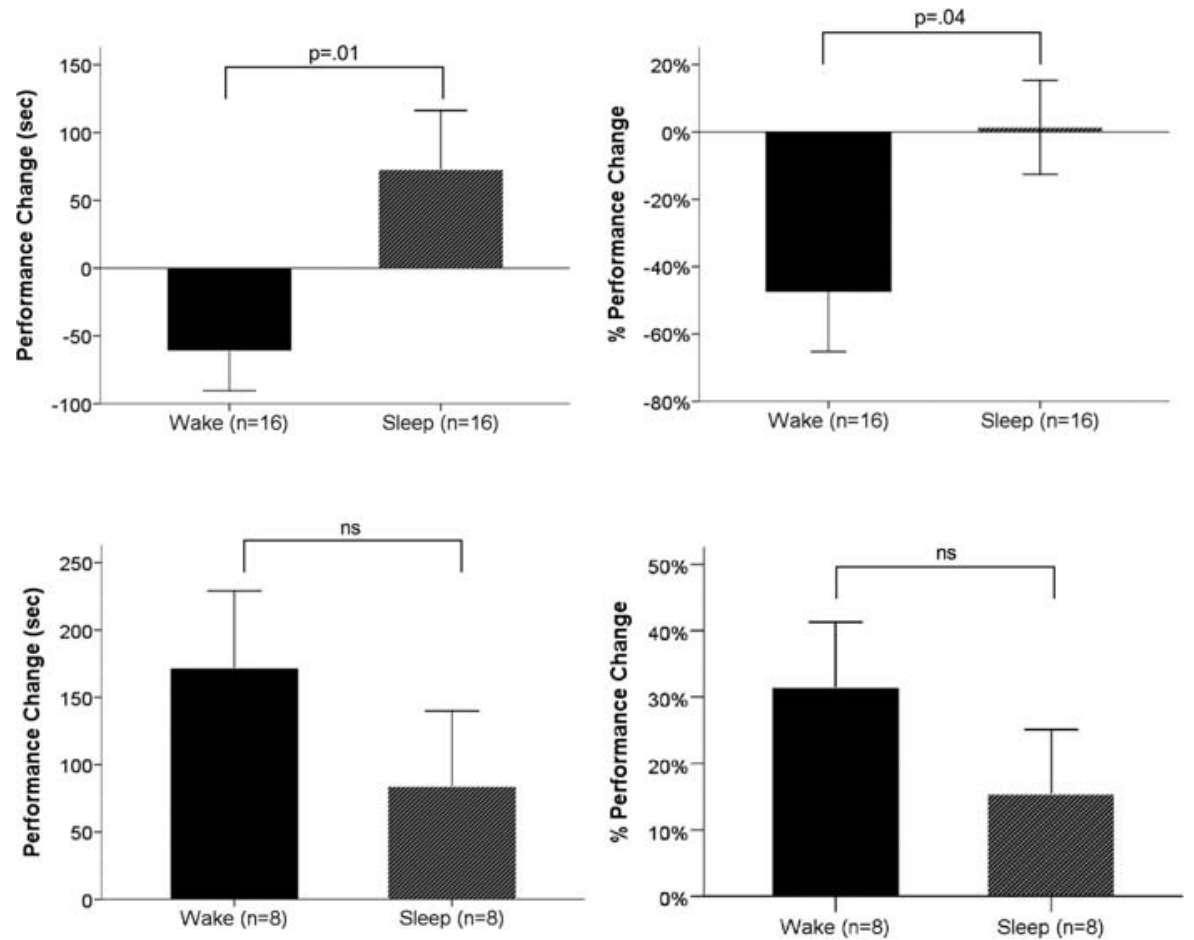

Figure 2. The effect of sleep on maze performance in Experienced (top) and Novice (bottom) game players. Performance changes are expressed as raw improvement (left) and percentage improvement (right) from last training trial. Error bars represent SEM. (ns) Nonsignificant. 

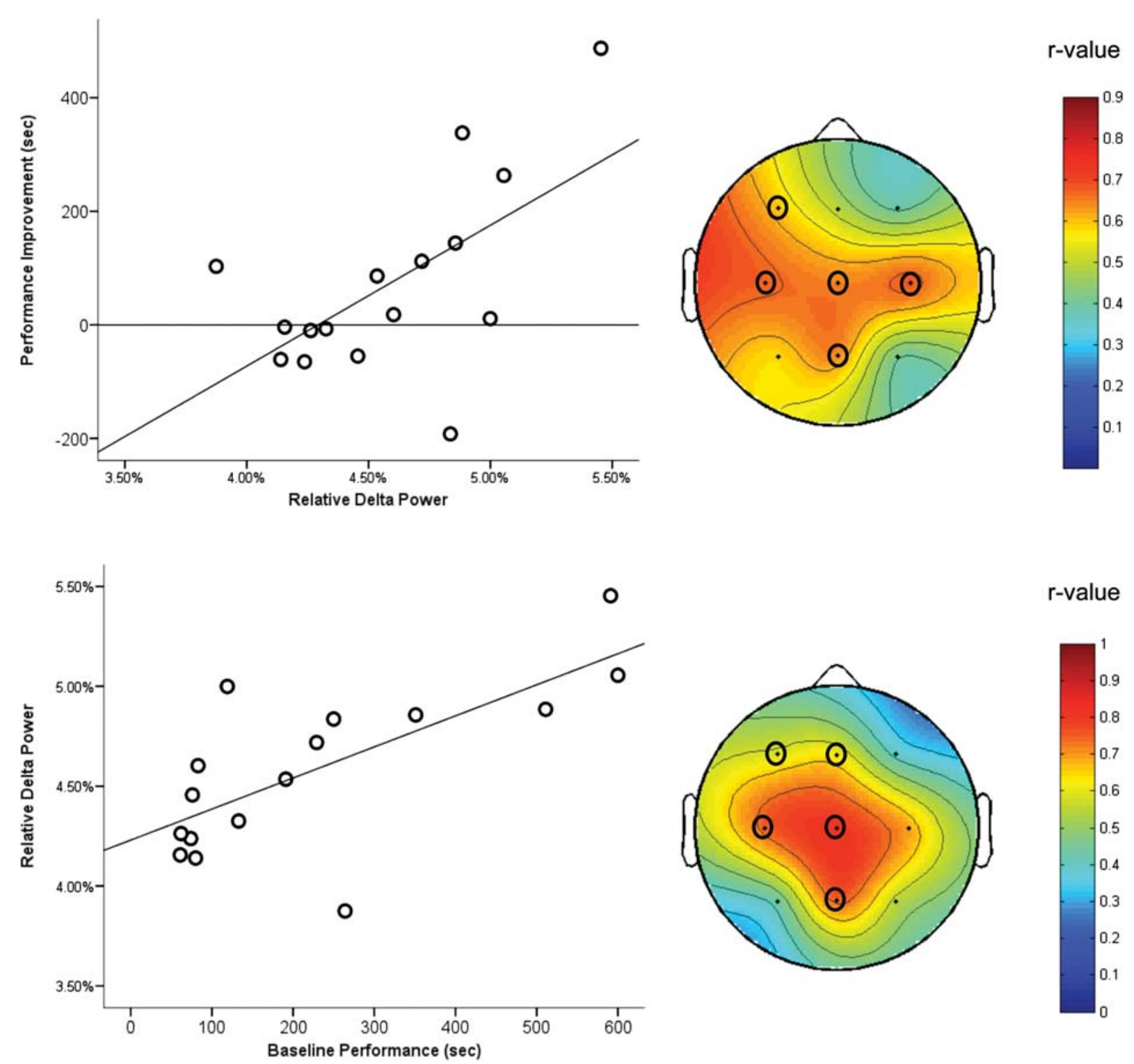

Figure 3. Performance and delta power. (Top left) Correlation between improvement from last training trial and mean delta power during stage 2 NREM in experienced players. (Bottom left) Correlation between baseline performance and mean delta power across all electrodes during stage 2 NREM sleep in experienced game players. Delta power is expressed as a percent of total power. (Right) Topographic plots depict the correlation between delta power and performance variables at individual electrodes. $(O)$ Indicates electrode cites which retain significance after correction for multiple comparisons.

deterioration on the task for experienced players. By design, the nap period was largely devoid of rapid eye movement (REM) sleep (see Table 1), demonstrating that a short period of NREM is sufficient to realize these sleep-dependent performance benefits.

The poor performance of novices at initial learning suggests that these subjects failed to adequately learn the route to the goal prior to sleep. In light of this poor baseline performance, it is perhaps not surprising that novices did not benefit from sleep, as prior research suggests that hippocampus-dependent tasks must be adequately encoded in order for sleep-dependent benefits to be realized (Peters et al. 2007; Tucker and Fishbein 2008). However, it could also be that performance improvements in novices differed qualitatively from those observed in experienced players. Novice players struggled with the motor/procedural aspects of the task, expressing difficulty and frustration with learning to use the keyboard to navigate through the maze, and often colliding with walls and other obstacles. Novices' improvement at retest may therefore have been procedural, relying on hippocampus-independent processes to support complex visuomotor skills required to move through the on-screen world. The consolidation of similar complex procedural skills has been demonstrated to depend selectively on REM sleep (Plihal and Born 1997; Smith 2001), while, in the present study, sleep subjects obtained only NREM sleep. As NREM sleep is thought to be particularly beneficial for hippocampal memory (Gais and Born 2004; Peigneux et al. 2004; Drosopoulos et al. 2007), we speculate that sleep could have stabilized route memory selectively in experienced players because only these subjects formed robust hippocampus-dependent spatial memory at training.

But what specific features of post-learning sleep account for the observed performance benefit in experienced players? Delta 
band (1-4 Hz) EEG activity in stage 2 NREM predicted improved performance at retest, with those subjects who exhibited the strongest stage 2 delta improving substantially (Fig. 3, top). Meanwhile, a robust correlation between baseline task performance and subsequent delta power (Fig. 3, bottom) suggests that the electrophysiological characteristics of nap sleep may themselves have been determined by subjects' presleep task performance. Previous studies have indeed demonstrated that intensive learning can lead to an augmentation of early night delta power (i.e., Huber et al. 2004), supporting the notion that increased delta during early nap sleep could have been directly induced by the challenging nature of the maze task. Alternatively, it could be that individuals with greater spatial navigation skill exhibit increased delta activity during this sleep stage. In either case, augmented low-frequency EEG power could support communication between the hippocampus and neocortex during post-learning NREM, at which time it is thought that the hippocampus mediates reactivation of learning-related neural networks, leading to the consolidation and reorganization of memories.

Taken together, these data suggest that sleep was beneficial for hippocampus-dependent route memory developed by experienced players during maze learning, protecting this recently formed spatial representation from the deleterious effects of decay and/or interference across the rest of the day. That memory performance was related to specific features of the sleep EEG, and selectively within experienced subjects, argues that an active sleep-specific process accounts for the observed effects. Further suggesting the presence of an active process during sleep, we observed that 20 min of quiet waking with reduced sensory interference was insufficient to prevent deterioration of route memory in the wake group, even though a much shorter period of sleep (6 min) has been shown to impart substantial performance benefits on a declarative memory task (Lahl et al. 2008). These observations suggest that the beneficial influence of the nap cannot be explained exclusively by a passive reduction of sensory input.

The present study contributes to a growing body of literature on hippocampus-dependent spatial memory and sleep, demonstrating that sleep confers a performance advantage for spatial navigation in humans. A large body of animal literature has clearly established that spatial exploration leads to reactivation of hippocampal place-cell networks during NREM (i.e., Wilson and McNaughton 1994; Lee and Wilson 2002; Ji and Wilson 2007) However, as "replay" of exploration-related network activity is typically assessed after intensive training on well-learned tasks, the potential contribution of this neuronal-level reactivation to beneficial effects on memory performance remains largely unknown. Here, post-learning sleep clearly led to a stabilization of route memory in humans. Although the present study cannot directly assess neuronal memory "reactivation," our data are consistent with the notion that recent learning experiences are processed "offline" during sleep, leading to improved post-sleep memory retention.

\section{Acknowledgments}

We thank Joseph Benavides and Mariette Asare for assistance with data collection, Masaki Nishida for assistance with EEG analyses, and Jon Chamberlain for his contributions to development of the virtual maze task. This research was supported by NIMH grant 48832 and NIH T32 training grant HL07901-10 to the Harvard Division of Sleep Medicine.

\section{References}

Astur RS, St Germain SA, Baker EK, Calhoun V, Pearlson GD, Constable RT. 2005. fMRI hippocampal activity during a virtual radial arm maze. Appl Psychophysiol Biofeedback 30: 307-317.
Backhaus J, Junghanns K. 2006. Daytime naps improve procedural motor memory. Sleep Med 7: 508-512.

Clemens Z, Fabo D, Halasz P. 2005. Overnight verbal memory retention correlates with the number of sleep spindles. Neuroscience 132: 529-535.

Clemens Z, Fabo D, Halasz P. 2006. Twenty-four hours retention of visuospatial memory correlates with the number of parietal sleep spindles. Neurosci Lett 403: 52-56.

Daurat A, Foret J, Bret-Dibat JL, Fureix C, Tiberge M. 2008. Spatial and temporal memories are affected by sleep fragmentation in obstructive sleep apnea syndrome. J Clin Exp Neuropsychol 30: 91-101.

De Gennaro L, Ferrara M. 2003. Sleep spindles: An overview. Sleep Med Rev 7: $423-440$.

Drosopoulos S, Windau E, Wagner U, Born J. 2007. Sleep enforces the temporal order in memory. PLoS One 2: e376. doi: 10.1371/ journal.pone.0000376.

Ellenbogen JM, Hulbert JC, Stickgold R, Dinges DF, Thompson-Schill SL. 2006. Interfering with theories of sleep and memory: Sleep, declarative memory, and associative interference. Curr Biol 16: 1290-1294.

Ferrara M, Iaria G, De Gennaro L, Guariglia C, Curcio G, Tempesta D, Bertini M. 2006. The role of sleep in the consolidation of route learning in humans: A behavioural study. Brain Res Bull 71: 4-9.

Ferrara M, Iaria G, Tempesta D, Curcio G, Moroni F, Marzano C, De Gennaro L, Pacitti C. 2008. Sleep to find your way: The role of sleep in the consolidation of memory for navigation in humans. Hippocampus 18: $844-851$.

Gais S, Born J. 2004. Declarative memory consolidation: Mechanisms acting during human sleep. Learn Mem 11: 679-685.

Huber R, Ghilardi MF, Massimini M, Tononi G. 2004. Local sleep and learning. Nature 430: 78-81.

Ji D, Wilson MA. 2007. Coordinated memory replay in the visual cortex and hippocampus during sleep. Nat Neurosci 10: 100-107.

Kudrimoti HS, Barnes CA, McNaughton BL. 1999. Reactivation of hippocampal cell assemblies: Effects of behavioral state, experience, and EEG dynamics. J Neurosci 19: 4090-4101.

Lahl O, Wispel C, Willigens B, Pietrowsky R. 2008. An ultra short episode of sleep is sufficient to promote declarative memory performance. J Sleep Res 17: 3-10.

Lee AK, Wilson MA. 2002. Memory of sequential experience in the hippocampus during slow wave sleep. Neuron 36: 1183-1194.

Mednick S, Nakayama K, Stickgold R. 2003. Sleep-dependent learning: A nap is as good as a night. Nat Neurosci 6: 697-698.

Milner B. 1965. Visually-guided maze learning in man: Effects of bilateral hippocampal, bilateral frontal and unilateral cerebral lesions. Neuropsychologia 3: 317-338.

Nadasdy Z, Hirase H, Czurko A, Csicsvari J, Buzsaki G. 1999. Replay and time compression of recurring spike sequences in the hippocampus. J Neurosci 19: 9497-9507.

Nishida M, Walker MP. 2006. Daytime naps, sleep spindles, and motor memory consolidation. In Abstract presented at the Society for Neuroscience Annual Meeting. Society for Neuroscience, Atlanta, GA.

Orban P, Rauchs G, Balteau E, Degueldre C, Luxen A, Maquet P, Peigneux P. 2006. Sleep after spatial learning promotes covert reorganization of brain activity. Proc Natl Acad Sci 103: 7124-7129.

Peigneux P, Laureys S, Fuchs S, Collette F, Perrin F, Reggers J, Phillips C, Degueldre C, Del Fiore G, Aerts J, et al. 2004. Are spatial memories strengthened in the human hippocampus during slow wave sleep? Neuron 44: $535-545$.

Peters KR, Smith V, Smith CT. 2007. Changes in sleep architecture following motor learning depend on initial skill level. J Cogn Neurosci 19: $817-829$.

Plihal W, Born J. 1997. Effects of early and late nocturnal sleep on declarative and procedural memory. J Cogn Neurosci 9: 534-547.

Plihal W, Born J. 1999. Effects of early and late nocturnal sleep on priming and spatial memory. Psychophysiology 36: 571-582.

Schabus M, Gruber G, Parapatics S, Sauter C, Klosch G, Anderer P, Klimesch W, Saletu B, Zeitlhofer J. 2004. Sleep spindles and their significance for declarative memory consolidation. Sleep 27: 1479-1485.

Smith C. 2001. Sleep states and memory processes in humans: Procedural versus declarative memory systems. Sleep Med Rev 5: 491-506.

Tucker MA, Fishbein W. 2008. Enhancement of declarative memory performance following a daytime nap is contingent on strength of initial task acquisition. Sleep 31: 1-7.

Tucker MA, Hirota Y, Wamsley EJ, Lau H, Chaklader A, Fishbein W. 2006. A daytime nap containing solely non-REM sleep enhances declarative but not procedural memory. Neurobiol Learn Mem 86: 241-247.

Wilson MA, McNaughton BL. 1994. Reactivation of hippocampal ensemble memories during sleep. Science 265: 676-679.

Received April 6, 2010; accepted in revised form April 29, 2010. 


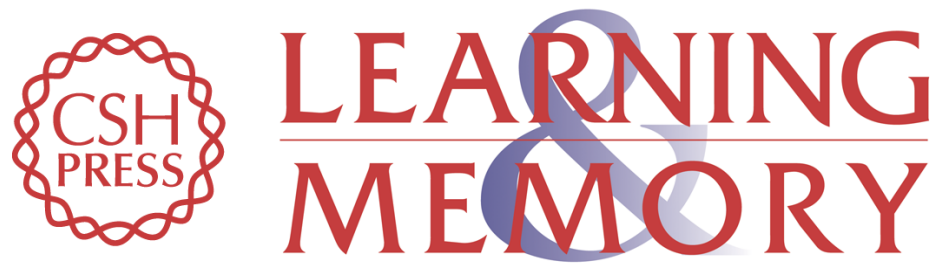

\section{A brief nap is beneficial for human route-learning: The role of navigation experience and EEG spectral power}

Erin J. Wamsley, Matthew A. Tucker, Jessica D. Payne, et al.

Learn. Mem. 2010, 17:

Access the most recent version at doi:10.1101//m.1828310

Supplemental
Material http://learnmem.cshlp.org/content/suppl/2010/06/25/17.7.332.DC1

References This article cites 29 articles, 5 of which can be accessed free at: http://learnmem.cshlp.org/content/17/7/332.full.html\#ref-list-1

License

Email Alerting Receive free email alerts when new articles cite this article - sign up in the box at the Service top right corner of the article or click here. 